Published in final edited form as:

Neurology. 2006 October 10; 67(7): 1120-1127. doi:10.1212/01.wnl.0000238514.51747.3a.

\title{
Treatment of neurocysticercosis:
}

\section{Current status and future research needs}

T.E. Nash, MD, G. Singh, MD, A.C. White, MD, V. Rajshekhar, MCh, J.A. Loeb, MD, PhD, J.V. Proaño, MD, O.M. Takayanagui, MD, A.E. Gonzalez, DVM, PhD, J.A. Butman, MD, PhD, C. DeGiorgio, MD, O.H. Del Brutto, MD, A. Delgado-Escueta, MD, PhD, C.A.W. Evans, MD, PhD, R.H. Gilman, MD, DTMH, S.M. Martinez, MD, M.T. Medina, MD, E.J. Pretell, MD, J. Teale, PhD, and H.H. Garcia, MD, PhD

Laboratory of Parasitic Diseases (T.E.N.), National Institute of Allergy and Infectious Diseases, $\mathrm{NIH}$, Bethesda, MD; Dayanand Medical College and Hospital (G.S.), Ludhiana, Punjab, India; Infectious Disease Section (A.C.W.), Department of Medicine, Baylor College of Medicine, Houston, TX; Department of Neurological Sciences (V.R.), Christian Medical College and Hospital, Vellore, India; Department of Neurology and Center for Molecular Medicine and Genetics (J.A.L.), Wayne State University School of Medicine, Detroit, MI; Medical Research Unit for Neurologic Diseases (J.V.P.), Hospital de Especialidades, Centro Medico Nacional Siglo XXI, Mexican Institute of Social Security, Mexico City; Department of Neurology (O.M.T.), School of Medicine of Ribeirao Preto, Universidade de Sao Paulo, Ribeirão Preto, Brazil; School of Veterinary Medicine (A.E.G.), Universidad Nacional Mayor de San Marcos, Lima, Perú; Department of International Health (A.E.G., R.H.G., H.H.G.), Johns Hopkins University Bloomberg School of Hygiene and Public Health, Baltimore, MD; Diagnostic Radiology Department (J.A.B.), Warren G. Magnuson Clinical Center, NIH, Bethesda, MD; Department of Neurology (C.D.G., A.D.-E.), Reed Neurological Research Center, UCLA, Los Angeles, CA; Department of Neurological Sciences (O.H.D.B.), Hospital-Clínica Kennedy, Guayaquil, Ecuador; VA Epilepsy Center of Excellence (A.D.-E.), Los Angeles, CA; University of Cambridge Clinical School (C.A.W.E.), Cambridge, and Department of Infectious Diseases and Microbiology, Imperial College London, London, UK; Department of Microbiology (R.H.G., E.J.P., H.H.G.), Universidad Peruana Cayetano Heredia, Lima, Perú; Cysticercosis Unit (S.M.M., H.H.G.), Instituto Especializado en Ciencias Neurológicas, Lima, Peru; Universidad Nacional Autonoma de Honduras (M.T.M.), Tegucigalpa, Honduras; and Department of Microbiology and Immunology (J.T.), University of Texas Health Science Center, San Antonio.

\section{Abstract}

\begin{abstract}
Here we put forward a roadmap that summarizes important questions that need to be answered to determine more effective and safer treatments. A key concept in management of neurocysticercosis is the understanding that infection and disease due to neurocysticercosis are variable and thus different clinical approaches and treatments are required. Despite recent advances, treatments remain either suboptimal or based on poorly controlled or anecdotal experience. A better understanding of basic pathophysiologic mechanisms including parasite survival and evolution, nature of the inflammatory response, and the genesis of seizures, epilepsy, and mechanisms of anthelmintic action should lead to improved therapies.
\end{abstract}

Copyright $@ 2006$ by AAN Enterprises, Inc.

Address correspondence and reprint requests to Dr. T.E. Nash, Laboratory of Parasitic Diseases, National Institute of Allergy and Infectious Diseases, NIH, Bethesda, MD; tnash@niaid.nih.gov.

Disclosure: The authors report no conflicts of interest. 
Cysticercosis, the most common cause of adult-onset epilepsy in the developing world, ${ }^{1}{ }^{3}$ is due to infection with the cystic larval form of the tapeworm Taenia solium. The intestinal dwelling tapeworm stage develops following the ingestion of raw or poorly cooked pork containing cystic larvae. The tapeworm releases infectious ova into the feces. When ingested by free-roaming pigs, the ova release the invasive larvae, which migrate and develop into cystic larvae in the muscles, brain, and other tissues of the pig. Like the pig, humans also develop larval cysts in their tissue after accidental ingestion of ova. Most of the symptoms and disease in humans result from infection of the CNS by larval cysts. Presenting symptoms and signs can be particularly varied due to differences in location, number of cysts, and associated inflammation. ${ }^{1,}$,

Treatment of neurocysticercosis (NCC) is controversial. ${ }^{1}{ }^{4}-9$ There are limited natural history studies of most types of NCC so that the benefits of treatments, until most recently, have not been based on optimally designed studies. Despite this imperfect supporting information, clinical observations indicate that treatment approaches and requirements differ among the forms or types of NCC. Factors considered in deciding treatments include the anatomic location of cysts, the stage of evolution of the cysts, number of cysts, the degree of associated inflammation, size, and severity of symptoms. Frequently, the presence of multiple cysts in different anatomic locations and in varying stages of evolution further complicates treatment decisions. Determining effective treatments is complicated by the fact that cysticidal treatment itself initiates a host inflammatory response that may result in the symptoms that one is trying to control.

Like many complex diseases that evolve over a long time, multiple types of treatments are used. ${ }^{4}$ Although treatment with the cysticidal agents albendazole and praziquantel has been the focus of a majority of reports, other treatments are also employed and important. These include use of corticosteroids and other immunosuppressive/anti-inflammatory medications to control damaging and potentially harmful host inflammatory responses, antiepileptic drugs (AEDs), surgical interventions, and general supportive measures. Although corticosteroids are commonly used to control signs and symptoms, their use has not been systematically studied. Short-term use is associated with transient side effects that are usually tolerated; however, long-term treatment is commonly required in complicated NCC, and side effects are frequent, severe, and even life threatening.

Most of our current treatments are based on empiric observations and subsequent refinements through clinical trials. We lack a scientific understanding of the pathophysiologic processes involved. Unraveling the basic mechanisms underlying the processes responsible for clinical disease expression and their interactions can be expected to lead to novel, improved, and safer treatments to prevent short-term complications and long-term sequelae. Three main basic processes take part in most of the disease and its consequences: 1) the biology of the cystic larva (growth, survival, degeneration, and death); 2) the host inflammation directed to the larva (induction, type, character, and role in pathogenesis and clearance of parasite material); and 3) the nature and character of brain damage and genesis of seizures and epilepsy.

How cysts develop, survive, grow, and degenerate is poorly understood. However, these are important factors that determine the nature and complications due to cysticercosis. Inflammation is a dominant if not pivotal process in disease pathogenesis of most forms of NCC. Seizures, the most common manifestation of NCC, somehow arise as a consequence of this inflammatory process. In addition, the latter is the starting point of a series of events that result in epilepsy. Knowing how seizures and epilepsy develop may lead to a better understanding of the pathogenesis of other forms of epilepsy and rational methods to control seizures in this population. A number of experts in parasitology, neurology, immunology, 
epilepsy, and veterinary medicine met last year to review the current status and issues in the treatment of NCC. The goal of the conference was to determine ways to improve the treatment of NCC by defining important questions and approaches to answer these questions. As the issues and problems associated with the frequently encountered different clinical types of cysticercosis are unique, they are discussed separately below.

\section{Intraparenchymal NCC}

\section{Viable cysts}

Cysts most commonly lodge in the parenchyma of the brain. Parenchymal cysts may be viable, degenerating (enhancing lesions), or already dead and calcified. ${ }^{10}$ Viable parenchymal cysts may be asymptomatic. However, they are more frequently seen in patients with seizures in endemic areas. Presumably, the viable cysts more frequently degenerate and initiate an injurious inflammatory response. Mass effects as well as focal neurologic symptoms and signs may also be caused by viable parenchymal cysts.

Many studies have documented that anthelmintic therapy results in death and resolution of viable cysts, ${ }^{5},{ }_{-} 9,11,{ }^{12}$ but the clinical benefit of this treatment had been questioned. ${ }^{6}$ As noted, treatment predictably injures cysts and initiates a sometimes intense inflammatory response to them ${ }^{13}$ that mimics the natural evolution of cysts. Seizures, headaches, and uncommonly death can occur secondary to cysticidal treatments. Until recently it had been unclear if it was better to kill cysts with anthelmintic agents and manage or prevent acute symptoms at the time of treatment or not to employ anthelmintics, then intervene and treat symptomatically as required. The recent publication of a randomized, placebocontrolled trial of treatment of adults with 20 or less viable parenchymal cysts and a history of seizures with albendazole and a short course of corticosteroids showed long-term clinical benefit and a decrease in generalized seizures and a nonsignificant decrease in all seizures following treatment. ${ }^{7}$ Although earlier randomized controlled studies indicated clinical benefit of treatment in some studies, ${ }^{7}-{ }^{9},{ }^{12}$ others failed to reach significance. ${ }^{6}$

A recent meta-analysis of these treatment studies confirmed that anthelmintic treatment of parenchymal NCC is clinically beneficial. ${ }^{14}$ This study and the reanalysis of prior studies shift the prevailing opinion from one of disputed clinical benefit to one of proven efficacy of treatment. The Garcia et al. multicystic treatment trial ${ }^{7}$ also found a correlation (although not significant) between the presence of residual viable cysts and the occurrence of seizures. A number of studies earlier found correlations between the presence of viable or degenerating cysts and increased risks of seizures. ${ }^{6},{ }^{15}{ }^{18}$ Though the Garcia et al. study is a good beginning in as much as it establishes the benefits of anthelmintic treatment of viable cysts, the magnitude of benefit demonstrated in the trial leaves much to be desired. For instance, it showed that only about half of the subjects irrespective of treatment remained seizure-free over a 2.5-year period, and only one-third of the subjects were cured of their disease as shown by imaging at 6 months. This means that more effective treatments are required to maximize clinical benefit and reduce morbidity due to seizures.

One of the diagnostic hallmarks of NCC is the presence viable, degenerating, and calcified cysts in the same individual. Although some cysts are the focus of intense inflammatory responses, others have no or little associated host responses and can remain undisturbed for long periods of time. Besides living in protected environments like brain and eye, mechanisms described to be associated with immune evasion in cysticercosis include complement inhibition, secretion of distractive antigens, cytokine expression, masking with host's immunoglobulins, among others. ${ }^{19},{ }^{20}$ Understanding how the parasite evades the host's responses and other host-parasite interactions may lead to novel, safer, and more effective interventions. Studies are beginning to understand potential mechanisms that 
prevent effective host responses to viable cysts ${ }^{21}$ and how inflammatory responses to cysts provoke seizures. ${ }^{22},{ }^{23}$ However, therapies specifically designed to modify or prevent the damaging inflammation caused by degenerating cysts have not yet been systematically evaluated.

\section{Questions and approaches concerning treatment of viable cysts}

Studies need to identify and evaluate more effective, safe, and efficient anthelmintic treatments and the safest and most effective accompanying anti-inflammatory agents. Evidence from current studies suggests that the amount of time a patient is exposed to viable cysts that can potentially degenerate or to currently degenerating cysts correlates with an increased risk of seizures. Therefore, methods to decrease the time at risk or to decrease the risk through judicious use of antiparasitic, antiinflammatory, or immunosuppressive treatments should be studied.

Although both praziquantel and albendazole are proven cysticidal agents, their effectiveness is variable, and not all cysts die after a single course of antiparasitic treatment. This needs to be improved with better regimens, better agents, or combinations of agents. Even though there is a significant reduction in numbers of cysts, others survive and increase the likelihood of seizures. The philosophy of treatment has been to use the least intervention in terms of time and amount of drug to obtain reasonable cure rates in a majority but not all patients. However, as the risk of seizures appears to correlate with the presence of viable and degenerating cysts, consideration should be given to a more individualized goal of eliminating all or almost all viable cysts where this approach is practical.

An understanding of the mechanisms of action of cysticidal medications may lead to development and use of safer and more effective drugs or drug combinations. Oxfendazole, a drug that has been shown to be particularly useful in the treatment of cysticercosis in swine, ${ }^{24}$ and other potentially anticysticidal drugs should be evaluated in humans. Operationally, the methods of the trial by Garcia et al. can serve for initial comparison to optimize therapy. ${ }^{7}$

The use of anti-inflammatory agents in cysticercosis has not been systemically studied, even though most experts favor the effectiveness of corticosteroids to one degree or another. ${ }^{4}$ If indeed seizures are associated with degenerating cysts and its associated inflammatory responses, then future research should address ways to prevent or minimize these inflammatory responses. These include studies specifically designed to understand 1) how the parasite remains viable and why there is no effective immune response; 2) what mechanisms are involved in initiation of the host immune response; 3) characterization of the immune response; 4) which aspects of the host response are involved in pathogenesis and which lead to parasite clearance; and 5) appropriate and safe methods to control inflammation associated with treatment-induced and naturally degenerating cysts.

Finally, studies need to address if and how treatment of viable cysts can lead to a decrease in epilepsy or seizures that is present in chronic cysticercosis, a later stage usually defined by the presence of dead calcified cysts. Recent studies indicate that most of the morbidity in NCC occurs in chronic cysticercosis. Studies also need to address the evolution and natural history of chronic cysticercosis and interventions to prevent and treat epilepsy associated with chronic disease.

\section{Single enhancing lesions}

A single degenerating cyst is the most frequent finding associated with NCC in the Indian subcontinent ${ }^{25}$ and is common in other regions as well. Degenerating cysts are usually associated with seizures because these cysts incite a granulomatous inflammatory response 
by the host. MRI of these lesions shows varying degrees of enhancement and edema, and histologic examination of these as well as nodular enhancing lesions (a more advanced stage of degeneration) invariably shows acute and or chronic granulomatous inflammation. ${ }^{26}{ }_{-}^{29}$

This type of presentation is the best studied of the clinical forms of NCC. Overall, there is a favorable prognosis. The effectiveness of antiparasitic treatments in this type of presentation has been variable. However, the most rigorous double-blinded randomized treatment trial showed an initial increase in seizures followed by a significant clinical benefit of anthelmintic treatment compared to antiseizure medication over the subsequent 2 years of evaluation. ${ }^{15},{ }^{30} \mathrm{~A}$ nonblinded follow-up evaluation of treated and nontreated subjects showed an equalization of seizure frequency and an overall good prognosis in both groups. ${ }^{15}$ As mentioned earlier, reanalysis of treatment trials of enhancing lesions ${ }^{30}{ }^{33}$ by metaanalysis showed an overall benefit of treatment. ${ }^{14}$ A potential reason for differences in individual study results is the heterogeneity of morphology of single enhancing lesions (SELs). Some SELs have a cystic or vesicular appearance, whereas others appear nodular or solid. Prior studies did not usually distinguish between these polar types of SELs. The cystic stage likely represents an early or nonresolving cyst in contrast to the solid nodular lesions that represent a more advanced state of degeneration and resolution. The former has been shown to resolve with anthelmintic treatment. ${ }^{26}$ Treatment trials comparing fully degenerated vs still cystic lesions may show more benefit of antiparasitic treatment in these selected patients. A recently published randomized trial comparing 14 days of prednisolone in the absence of anthelmintic therapy with placebo documented a decrease in seizure frequency in favor of prednisolone. ${ }^{34}$ However, another randomized open label trial found that prednisolone was significantly inferior in decreasing seizures compared with albendazole and prednisolone. ${ }^{18}$ Although treatment with albendazole and prednisolone may be superior to corticosteroid use alone, the former study ${ }^{34}$ is the best available information that judicious use of antiinflammatory agents are beneficial in decreasing seizures. ${ }^{34}, 35$

\section{Questions and approaches concerning treatment of SELs}

Although the long-term prognosis of these patients is relatively good with about $85 \%$ remaining seizure-free a mean of 65 months, in the absence of AEDs, $15 \%$ have recurrent seizures. Risk factors for recurrence are two or more seizures, breakthrough seizures, and residual calcification ${ }^{16}$ or other lesions. ${ }^{15}{ }_{-} 18$

SEL is one variety of NCC that is ideal for study because the natural history is well described and its involution unaltered by the potential modifying influences of additional parenchymal or extraparenchymal lesions. Given their frequency, measures to prevent the development of calcifications and chronic epilepsy are important. Last, although the followup and prognosis are known for about 5 years, this is a relatively short time in the life of these patients.

\section{Chronic calcific cysticercosis}

Eventually most parasites calcify, commonly appearing as punctate hyperdensities on CT examinations. Brain calcifications typical of NCC are detected in from 15 to $20 \%$ of endemic populations. ${ }^{36}{ }^{41}$ Calcifications in the absence of viable cysts are frequently the only or predominant type of lesion found in patients who present with seizures and have been implicated as seizure foci in studies. As treatment of viable cysts results in a decrease in the seizure rate, calcified and resolved cysts necessarily cause seizures at a lower rate than viable and degenerating cysts. However, the number of individuals with calcifications is large, ${ }^{36}{ }_{-}^{41}$ and even though there is a relatively low frequency of seizures, there are a considerable number of affected persons. 
Previously, calcific NCC was characterized as "inactive," implying it was not associated with significant disease or morbidity. Recently, perilesional edema around calcified lesions has been detected around foci of seizure activity or focal neurologic deficits in symptomatic patients (figure 1) ${ }^{42}{ }^{44}$ How calcified cysticerci cause seizures or focal neurologic symptoms is not understood. Although the overall importance and pathogenesis of this phenomenon have not been well defined, preliminary evidence suggests episodic perilesional edema is common in this population. ${ }^{44}$ In addition, the presence of enhancement around calcified lesions and the periodic presence of edema are suggestive of an inflammatory etiology, perhaps due to sporadic release of antigen and subsequent host immunologic responses. ${ }^{42}{ }_{-} 45$

\section{Questions and major approaches}

Chronic cysticercosis as an important cause of seizures has only recently been appreciated, and consequently a reasonable understanding of its importance and knowledge of its pathogenesis are lacking. Prospective studies are required to determine the natural history, range of clinical manifestations, seizure frequency, and how best to prevent and treat associated seizures in this group. A subset of patients presents with perilesional edema that can be frequent, recurrent, and life altering. The pathogenesis and whether the edema represents an inflammatory process or a response to focal epileptic activities are not known. A vexing question is why only a few of many lesions in the same individual are associated with perilesional edema and symptoms. There is no proven effective therapy so trials of prevention and treatment are needed. A reasonable hypothesis is that prior inflammation somehow generates a seizure focus. Enhanced measures to control inflammation or the resulting calcification induced by degenerating cysts may result in decreased rates of seizures in chronic calcific cysticercosis. It has recently been suggested that intraparenchymal calcifications alone can be toxic and promote seizures and headaches that can be reversed by calcium chelation with bisphosphonates. ${ }^{46}$ The association of NCC with mesial temporal lobe epilepsy due to hippocampal sclerosis may provide new insights into epileptogenesis. ${ }^{47}, 48$

\section{Extraparenchymal NCC}

Extraparenchymal cysticercosis refers primarily to infection of the subarachnoid spaces and ventricles. The clinical problems, approaches, and treatment differ from parenchymal infection, and generally, the prognosis of these patients is worse compared with those with solely parenchymal disease. ${ }^{49}$ Cysts may be small or large; the latter commonly present with lifethreatening mass lesions. ${ }^{49}$ They may or may not regress after anthelmintic treatment and in contrast to parenchymal cysts frequently require long-term and multiple courses of anthelmintics. ${ }^{50}$ Meningeal inflammation is common and may lead to lacunar infarcts and hydrocephalus requiring placement of intraventricular shunts. However, shunt infection and failure are common..$^{51}$ There is a subset of patients who manifest persistent inflammation despite prolonged if not heroic courses of treatment and seem to require continuous antiinflammatory medication; most importantly, there are no controlled studies of treatment.

\section{Subarachnoid cysts}

A subset of subarachnoid located cysts grows abnormally, presenting as large mass occupying lesions or as racemose cysts, 52 which are aberrantly proliferating membranous forms (figure 2). Giant cysts respond to anthelmintic drugs. Inflammatory responses to subarachnoid and ventricular cysts frequently cause chronic meningitis and ependymitis that in turn result in hydrocephalus, a common long-term complication, necessitating shunt placement. Lacunar infarcts and entrapment of cranial nerves occur as a result of granulomatous inflammation affecting adjacent arteries or nerves. Whether inflammation in 
chronic cysticercosis meningitis is the result of continuous degeneration or chronic antigenic release from dead parasitic tissues/ cells driving host inflammatory responses is not known. Inhibition of maturation, growth, and abnormal proliferation forms would be expected to be effective treatments and result in better clinical outcomes.

\section{Inflammation in subarachnoid cysticercosis}

The importance of inflammation as a cause of morbidity and mortality is the most direct and obvious in subarachnoid and ventricular cysticercosis both from clinical observations and from autopsy examinations. Yet, very little is known about the nature of the inflammation and safe and effective measures to control it. The genesis of chronic inflammation despite long-term anthelmintic treatment is not known. There are several potential contributors. First, CSF levels of both praziquantel and albendazole sulfoxide are erratic and influenced by other medications ${ }^{53}$ so that inadequate CSF drug levels may lead to continued parasite growth and degeneration that likely results in continued antigen release and subsequent host inflammatory responses. Second, parasite materials may not be easily processed in the subarachnoid space. Third, anthelmintic treatment may not be fully effective in the presence of immunosuppressive medications. Other larval cestodes are capable of dissemination in immunosuppressed humans or animals so that the idea of persons being chronically immunosuppressed permitting some proliferation and antigen release is possible but currently without supporting clinical data.

\section{Surgical choices}

Surgery in subarachnoid NCC is mostly restricted to shunt placement or emergency decompression. In the presence of acute obstructive hydrocephalus, surgical therapy can be lifesaving. In many cases, this can be accomplished by placement of a ventricle-peritoneal shunt. Case series suggest that shunt failure rates are high. However, the failure rate is lower in cases treated with antiparasitic drugs. As viable cysts can mechanically obstruct or degenerate and obstruct, surgical removal of cysts when feasible and safe seems a reasonable approach. On the other hand, several authors have reported successful antihelmintic treatment of giant subarachnoid cysts. ${ }^{50}, 54$ Additionally, surgery has inherent additional risks.

\section{Questions and major approaches}

Issues such as the roles and uses of anthelmintics and antiinflammatory agents singly and in combination need to be assessed in subarachnoid and ventricular cysticercosis. Variables such as degree of drug absorption and CSF levels, degree of immunosuppression, and assessment of parasite presence/growth need to be controlled or measured to understand possible roles in clinical outcome variability. Taenia antigens have been detected in serum and CSF of certain types of NCC. ${ }^{55} 58$ The presence and amount of Taenia antigen in the CSF or serum may correlate with presence and growth of subarachnoid cysts and be particularly helpful in assessing presence, growth, and effectiveness of treatments. ${ }^{59}$ The practical usefulness of these tests needs to be shown and, if there is utility, assays standardized and made generally available. Subarachnoid or ventricular NCC is relatively uncommon, and trials should require multicenter participation to obtain enough patients. Standardization of treatments for comparison is required.

Basic studies are needed to define the nature of the inflammatory process invoked by extraparenchymal larval cysts. Animal models using other cestodes, the infected pig, and human studies can be used to determine the characteristics of the inflammation and what agents can safely control the inflammation over the time period required. In addition, the role of continued parasite regeneration or metabolism needs to be assessed using longer and shorter anthelmintic regimens in conjunction with immunosuppressive agents. Methotrexate 
has recently been reported to be an effective replacement or corticosteroid-sparing agent for long-term control of meningeal inflammation. ${ }^{60}$ The development of effective and novel biologic immunomodulatory agents offers the possibility of relatively specific and safer antiinflammatory agents.

Given that anthelmintic treatments usually initiate an inflammatory response that commonly requires immunosuppression, one strategy that could be of benefit is surgical removal of approachable lesions that are large, likely to lead to long-term exposure to immunosuppressives or anthelmintics, or to prevent complications. For instance, surgical removal of a large 3-cm-diameter cerebellar cyst encroaching on the fourth ventricle in the presence of normal-sized multiple parenchymal cysts would be expected to minimize the possible complications of medical treatment and the duration of therapy. The morbidity and mortality related to surgery would likely be less than the complications of long-term immunosuppression and complications from a shunt, if it were required. How, when, and which cysts should be removed should be determined. Presumably cysts showing little accompanying inflammation or viable or unattached to the surrounding structures can be removed with minimal damage to critical areas.

\section{Ventricular disease}

Cysts that lodge in the ventricles may result in hydrocephalus by blocking CSF flow mechanically or as a result of inflammation and fibrosis. Blockage of the fourth ventricle and to a lesser extent third ventricle is relatively frequent and gives rises to the most serious complications. Cysts located in the lateral ventricles can cause unilateral hydrocephalus but may also resolve without sequelae. Hydrocephalus is a common complication requiring placement of intraventricular shunts. There are no randomized treatment trials comparing the usefulness and safety of medical compared with surgical approaches.

\section{Surgical choices}

Surgery is mostly restricted to shunt placement or removal of cysts located in the ventricles. One series has shown that anthelmintic treatment of fourth ventricular cysts ${ }^{61}$ can be effective. There are a number of convincing reports documenting the use of less invasive flexible neuroendoscopy to remove approachable subarachnoid cysts and cysts lodged in the lateral, third, and fourth ventricles. ${ }^{62}{ }^{65}$ These reports suggest that in the hands of trained neurosurgeons, this method may be safer, faster, and perhaps more definitive and subsequently may lead to better long-term clinical outcomes. Most intraventricular cysts seem to be freely floating and therefore may be extracted without trauma to the brain and avoid inflammation that would be elicited by anthelmintic therapy

\section{Questions and major approaches}

The role of anthelmintic therapy in ventricular cysts has not been adequately studied, although medical treatment is commonly suboptimal. It is likely that both medical and surgical treatments are useful in ventricular NCC, but the conditions where each is best need to be defined. Trials comparing medical, surgical, or medical-surgical approaches are required. Important factors modifying approaches possibly include viability and location of the cyst, the urgency of the need for decompression, degree of accompanying inflammation and adherence to vital structures, and response to anti-inflammatory measures. Similar to subarachnoid disease, there are a few reports suggesting that anti-inflammatory agents such as corticosteroids and antiparasitic drugs are useful in preservation of intraventricular shunts. However, randomized trials, perhaps employing differing agents or regimens, are needed. 


\section{Seizure genesis}

In recent studies, NCC is the cause of about one-third of all epilepsy and seizure cases in endemic areas. The risk of seizures is greatest in disease associated with viable or degenerating cysts but continues to occur to a lesser extent in chronic calcific cysticercosis. Little is known about why and how seizures and epilepsy develop in this infection. There is reasonable information implicating host inflammatory responses. First, seizure foci commonly localize to inflamed or degenerating cysts. Histologic examination reveals surrounding inflammation, and concordant MRI studies demonstrate varying degrees of contrast enhancement or edema in contrast to viable cysts that show little or no inflammation by histologic examination or by MRI. This would be consistent with a recent study suggesting that disruption of the blood-brain barrier with leakage of serum components into the brain is sufficient to induce seizures. ${ }^{66}$ Second, anthelmintic treatment initiates a host inflammatory response around cysts that mimics histologic and radiologic findings typical of naturally degenerating cysts. Both are associated with an increased risk of seizures. Third, in a small animal model, seizures were induced by the host response rather than the parasite itself. ${ }^{23}$ The rational for using corticosteroids during treatment is an attempt to control the inflammation and accompanying seizures.

\section{Antiseizure medications}

Antiseizure medication is indicated in all patients with epilepsy and most with seizures. Although there is no information suggesting that use of AEDs in NCC differs from use in other similar types of epilepsy and seizures, this has not been formally studied. In addition, recent studies indicate that some AEDs may affect transcription through alteration of histones. ${ }^{67},{ }^{68}$ Theoretically, they may affect the cyst directly and have effects on course of treatment and even disease.

\section{Questions and major approaches}

As emphasized above, NCC is a common cause of seizures and epilepsy in endemic populations. Because cysticercosis is an acquired cause of epilepsy permitting prospective studies, seizure foci are commonly identifiable, and active and inactive foci can be directly compared in the same patient, these patients are uniquely valuable to study the pathogenesis of epilepsy. Inflammation likely plays a pivotal role in the genesis of seizures and epilepsy. Use of model infections in pigs for instance and sophisticated neuroimaging studies in afflicted patients can be expected to yield an understanding of important basic processes.

\section{General recommendations}

There was overall agreement in support of the following general recommendations: 1) support by international health organizations for the development for multicenter clinical trials; 2) organization of multidisciplinary treatment units; 3) recognition that understanding of basic pathophysiologic mechanisms including survival, growth, and degeneration of the parasite, nature and character of the inflammatory response, and the genesis of seizures, epilepsy, and mechanisms of anthelmintic action will lead to safer, specific, and more effective therapies; and 4) development of noninvasive neuroimaging to assess the nature and degree of inflammation and location and state of the larval cestodes and neuroexcitation. NCC continues to be a major cause of neurologic disease in most of the world. Despite the importance and associated burden of disease (mainly related to chronic, many times disabling neurologic symptoms), key pieces of knowledge are still missing, hampering attempts to optimize clinical care. 


\section{Acknowledgments}

Supported by the Office of Rare Diseases (NIH), National Institute of Allergy and Infectious Diseases/Laboratory of Parasitic Diseases (NIH), Universidad Peruana Cayetano Heredia (Lima, Peru), and Instituto de Ciencias Neurologicas (Lima, Peru).

\section{References}

1. Grcia HH, Del Brutto OH. Neurocysticercosis: updated concepts about an old disease. Lancet Neurol. 2005; 4:653-661. [PubMed: 16168934]

2. Commission on Tropical Diseases of the International League Against Epilepsy. Relationship between epilepsy and tropical diseases. Commission on Tropical Diseases of the International League Against Epilepsy. Epilepsia. 1994; 35:89-93. [PubMed: 8112262]

3. White AC Jr. Neurocysticercosis: a major cause of neurological disease worldwide. Clin Infect Dis. 1997; 24:101-114. [PubMed: 9114131]

4. Nash TE. Human case management and treatment of cysticercosis. Acta Trop. 2003; 87:61-69. [PubMed: 12781379]

5. Sotelo J, del Brutto OH, Penagos P, Escobedo F, Torres B, Rodriguez-Carbajal J, et al. Comparison of therapeutic regimen of anticysticercal drugs for parenchymal brain cysticercosis. J Neurol. 1990; 237:69-72. [PubMed: 2192018]

6. Carpio A, Santillan F, Leon P, Flores C, Hauser WA. Is the course of neurocysticercosis modified by treatment with antihelminthic agents? Arch Intern Med. 1995; 155:1982-1988. [PubMed: 7575052]

7. Garcia HH, Pretell EJ, Gilman RH, et al. A trial of antiparasitic treatment to reduce the rate of seizures due to cerebral cysticercosis. N Engl J Med. 2004; 350:249-258. [PubMed: 14724304]

8. Alarcon F, Dueñas G, Diaz M, Cevallos N, Estrada G. Short course of albendazole therapy for neurocysticercosis: a prospective randomized trial comparing three days, eight days and the control group without albendazole. Rev Ecuat Neurol. 2001; 10:1-6.

9. Sotelo J, Penagos P, Escobedo F, Del Brutto OH. Short course of albendazole therapy for neurocysticercosis. Arch Neurol. 1988; 45:1130-1133. [PubMed: 3178533]

10. Garcia HH, Del Brutto OH. Imaging findings in neurocysticercosis. Acta Trop. 2003; 87:71-78. [PubMed: 12781380]

11. Del Brutto OH, Santibanez R, Noboa CA, Aguirre R, Diaz E, Alarcon TA. Epilepsy due to neurocysticercosis: analysis of 203 patients. Neurology. 1992; 42:389-392. [PubMed: 1736171]

12. Vazquez V, Sotelo J. The course of seizures after treatment for cerebral cysticercosis. N Engl J Med. 1992; 327:696-701. [PubMed: 1495522]

13. Spina-Franca A, Nobrega JP, Livramento JA, Machado LR. Administration of praziquantel in neurocysticercosis. Tropenmed Parasitol. 1982; 33:1-4. [PubMed: 7101435]

14. Del Brutto OH, Roos KL, Coffey CS, García HH. Meta-analysis: cysticidal drugs for neurocysticercosis: albendazole and praziquantel. Ann Intern Med. 2006; 145:43-51. [PubMed: 16818928]

15. Baranwal AK, Singhi PD, Singhi SC, Khandelwal N. Seizure recurrence in children with focal seizures and single small enhancing computed tomographic lesions: prognostic factors on longterm follow-up. J Child Neurol. 2001; 16:443-445. [PubMed: 11417612]

16. Rajshekhar V, Jeyaseelan L. Seizure outcome in patients with a solitary cerebral cysticercus granuloma. Neurology. 2004; 62:2236-2240. [PubMed: 15210888]

17. Pradhan S, Kathuria MK, Gupta RK. Perilesional gliosis and seizure outcome: a study based on magnetization transfer magnetic resonance imaging in patients with neurocysticercosis. Ann Neurol. 2000; 48:181-187. [PubMed: 10939568]

18. Singhi P, Jain V, Khandelwal N. Corticosteroids versus albendazole for treatment of single small enhancing computed tomographic lesions in children with neurocysticercosis. J Child Neurol. 2004; 19:323-327. [PubMed: 15224704] 
19. Flisser, A.; Correa, D.; Evans, CAW. Taenia solium cysticercosis: new and revisited immunological aspects. In: Singh, G.; Prabhakar, S., editors. Taenia solium cysticercosis. From basic to clinical science. CABI Publishing; Oxon: 2002. p. 15-24.

20. White AC Jr, Robinson P, Kuhn R. Taenia solium cysticercosis: hostparasite interactions and the immune response. Chem Immunol. 1997; 66:209-230. [PubMed: 9103671]

21. Baig S, Damian RT, Morales-Montor J, Olecki P, et al. Characterization of excretory/secretory endopeptidase and metallo-aminopeptidases from Taenia crassiceps metacestodes. J Parasitol. 2005; 91:983-987. [PubMed: 16419737]

22. Robinson P, White AC, Lewis DE, Thornby J, David E, Weinstock J. Sequential expression of the neuropeptides substance $\mathrm{P}$ and somatostatin in granulomas associated with murine cysticercosis. Infect Immun. 2002; 70:4534-4538. [PubMed: 12117965]

23. Stringer JL, Marks LM, White AC Jr, Robinson P. Epileptogenic activity of granulomas associated with murine cysticercosis. Exp Neurol. 2003; 183:532-536. [PubMed: 14552894]

24. Gonzales AE, Garcia HH, Gilman RH, Gavidia CM, Tsang VC, Bernal T, et al. Effective, singledose treatment or porcine cysticercosis with oxfendazole. Am J Trop Med Hyg. 1996; 54:391-394. [PubMed: 8615453]

25. Rajshekhar V. Etiology and management of single small CT lesions in patients with seizures: understanding a controversy. Acta Neurol Scand. 1991; 84:465-470. [PubMed: 1792850]

26. Rajshekhar V. Albendazole therapy for persistent, solitary cysticercus granulomas in patients with seizures. Neurology. 1993; 43:1238-1240. [PubMed: 8170573]

27. Chandy MJ, Rajshekhar V, Ghosh S, Prakash S, Joseph T, Abraham J, et al. Single small enhancing CT lesions in Indian patients with epilepsy: clinical, radiological and pathological considerations. J Neurol Neurosurg Psychiatry. 1991; 54:702-705. [PubMed: 1940942]

28. Martinez HR, Rangel-Guerra R, Arredondo-Estrada JH, Marfil A, Onofre J. Medical and surgical treatment in neurocysticercosis a magnetic resonance study of 161 cases. J Neurol Sci. 1995; 130:25-34. [PubMed: 7650528]

29. Suss RA, Maravilla KR, Thompson J. MR imaging of intracranial cysticercosis: comparison with CT and anatomopathologic features. AJNR Am J Neuroradiol. 1986; 7:235-242. [PubMed: 3082155]

30. Baranwal AK, Singhi PD, Khandelwal N, Singhi SC. Albendazole therapy in children with focal seizures and single small enhancing computerized tomographic lesions: a randomized, placebocontrolled, double blind trial. Pediatr Infect Dis J. 1998; 17:696-700. [PubMed: 9726343]

31. Gogia S, Talukdar B, Choudhury V, Arora BS. Neurocysticercosis in children: clinical findings and response to albendazole therapy in a randomized, double-blind, placebo-controlled trial in newly diagnosed cases. Trans R Soc Trop Med Hyg. 2003; 97:416-421. [PubMed: 15259471]

32. Padma MV, Behari M, Misra NK, Ahuja GK. Albendazole in single CT ring lesions in epilepsy. Neurology. 1994; 44:1344-1346. [PubMed: 8035946]

33. Kalra V, Dua T, Kumar V. Efficacy of albendazole and short-course dexamethasone treatment in children with 1 or 2 ring-enhancing lesions of neurocysticercosis: a randomized controlled trial. J Pediatr. 2003; 143:111-114. [PubMed: 12915835]

34. Garg RK, Potluri N, Kar AM, et al. Short course of prednisolone in patients with solitary cysticercus granuloma: a double blind placebo controlled study. J Infect. 2005; 53:65-69. [PubMed: 16269179]

35. Mall RK, Agarwal A, Garg RK, Kar AM, Shukla R. Short course of prednisolone in Indian patients with solitary cysticercus granuloma and new-onset seizures. Epilepsia. 2003; 44:13971401. [PubMed: 14636346]

36. Cruz ME, Schantz PM, Cruz I, Espinosa P, Preux PM, Cruz A, et al. Epilepsy and neurocysticercosis in an Andean community. Int J Epidemiol. 1999; 28:799-803. [PubMed: 10480714]

37. Garcia-Noval J, Allan JC, Fletes C, Moreno E, DeMata F, TorresAlvarez R, et al. Epidemiology of Taenia solium taeniasis and cysticercosis in two rural Guatemalan communities. Am J Trop Med Hyg. 1996; 55:282-289. [PubMed: 8842116]

38. Medina MT, Duron RM, Martinez L, et al. Prevalence, incidence, and etiology of epilepsies in rural Honduras: the Salama Study. Epilepsia. 2005; 46:124-131. [PubMed: 15660778] 
39. Sanchez AL, Lindback J, Schantz PM, et al. A population-based, casecontrol study of Taenia solium taeniasis and cysticercosis. Ann Trop Med Parasitol. 1999; 93:247-258. [PubMed: 10562826]

40. Del Brutto OH, Santibanez R, Idrovo L, et al. Epilepsy and neurocysticercosis in Atahualpa: a door-to-door survey in rural coastal Ecuador. Epilepsia. 2005; 46:583-587. [PubMed: 15816956]

41. Montano SM, Villaran MV, Ylquimiche L, et al. Neurocysticercosis: association between seizures, serology, and brain CT in rural Peru. Neurology. 2005; 65:229-233. [PubMed: 16043791]

42. Nash TE, Del Brutto OH, Butman JA, et al. Calcific neurocysticercosis and epileptogenesis. Neurology. 2004; 62:1934-1938. [PubMed: 15184592]

43. Nash TE, Patronas NJ. Edema associated with calcified lesions in neurocysticercosis. Neurology. 1999; 53:777-781. [PubMed: 10489040]

44. Nash TE, Pretell J, Garcia HH. Calcified cysticerci provoke perilesional edema and seizures. Clin Infect Dis. 2001; 33:1649-1653. [PubMed: 11595994]

45. Sheth TN, Pillon L, Keystone J, Kucharczyk W. Persistent MR contrast enhancement of calcified neurocysticercosis lesions. AJNR Am J Neuroradiol. 1998; 19:79-82. [PubMed: 9432161]

46. Loeb JA, Sohrab SA, Huq M, Fuerst DR. Brain calcifications induce neurological dysfunction that can be reversed by a bone drug. J Neurol Sci. 2006; 243:77-81. [PubMed: 16430923]

47. Leite JP, Terra-Bustamante VC, Fernandes RM, et al. Calcified neurocysticercotic lesions and postsurgery seizure control in temporal lobe epilepsy. Neurology. 2000; 55:1485-1491. [PubMed: 11094102]

48. Wichert-Ana L, Velasco TR, Terra-Bustamante VC, et al. Surgical treatment for mesial temporal lobe epilepsy in the presence of massive calcified neurocysticercosis. Arch Neurol. 2004; 61:1117-1119. [PubMed: 15262746]

49. Estanol B, Corona T, Abad P. A prognostic classification of cerebral cysticercosis: therapeutic implications. J Neurol Neurosurg Psychiatry. 1986; 49:1131-1134. [PubMed: 3783174]

50. Proano JV, Madrazo I, Avelar F, Lopez-Felix B, Diaz G, Grijalva I. Medical treatment for neurocysticercosis characterized by giant subarachnoid cysts. N Engl J Med. 2001; 345:879-885. [PubMed: 11565520]

51. Sotelo J, Marin C. Hydrocephalus secondary to cysticercotic arachnoiditis. A long-term follow-up review of 92 cases. J Neurosurg. 1987; 66:686-689. [PubMed: 3572494]

52. Bickerstaff ER, Cloake PCP, Hughes B, Smith WT. The racemose form of cerebral cysticercosis. Brain. 1952; 75:1-16. [PubMed: 14916052]

53. Takayanagui OM. Therapy for neurocysticercosis. Expert Rev Neurother. 2004; 4:129-139. [PubMed: 15853623]

54. Del Brutto OH. Albendazole therapy for subarachnoid cysticerci: clinical and neuroimaging analysis of 17 patients. J Neurol Neurosurg Psychiatry. 1997; 62:659-661. [PubMed: 9219761]

55. Estrada JJ, Kuhn RE. Immunochemical detection of antigens of larval Taenia solium and antilarval antibodies in the cerebrospinal fluid of patients with neurocysticercosis. J Neurol Sci. 1985; 71:39-48. [PubMed: 4087018]

56. Correa D, Sandoval MA, Harrison LJ, et al. Human neurocysticercosis: comparison of enzyme immunoassay capture techniques based on monoclonal and polyclonal antibodies for the detection of parasite products in cerebrospinal fluid. Trans R Soc Trop Med Hyg. 1989; 83:814-816. [PubMed: 2694513]

57. Garcia HH, Harrison LJ, Parkhouse RM, et al. A specific antigendetection ELISA for the diagnosis of human neurocysticercosis. The Cysticercosis Working Group in Peru. Trans R Soc Trop Med Hyg. 1998; 92:411-414. [PubMed: 9850394]

58. Fleury A, Hernandez M, Fragoso G, Parkhouse RM, Harrison LJ, Sciutto E. Detection of secreted cysticercal antigen: a useful tool in the diagnosis of inflammatory neurocysticercosis. Trans R Soc Trop Med Hyg. 2003; 97:542-546. [PubMed: 15307421]

59. Zamora H, Castillo Y, Garcia HH, Pretall EJ, et al. Drop in antigen levels following successful treatment of subarachnoid neurocysticercosis. Am J Trop Med Hyg. 2005; 73:S41.

60. Keiser PB, Nash TE. Prolonged perilesional edema after treatment of parenchymal neurocysticercosis: methotrexate as a corticosteroid- sparing agent. Clin Infect Dis. 2003; 36:e122-e126. [PubMed: 12746791] 
61. Proano JV, Madrazo I, Garcia L, Garcia-Torres E, Correa D. Albendazole and praziquantel treatment in neurocysticercosis of the fourth ventricle. J Neurosurg. 1997; 87:29-33. [PubMed: 9202261]

62. Anandh B, Mohanty A, Sampath S, Praharaj SS, Kolluri S. Endoscopic approach to intraventricular cysticercal lesions. Minim Invasive Neurosurg. 2001; 44:194-196. [PubMed: 11830776]

63. Bergsneider M, Holly LT, Lee JH, King WA, Frazee JG. Endoscopic management of cysticercal cysts within the lateral and third ventricles. J Neurosurg. 2000; 92:14-23. [PubMed: 10616077]

64. Bergsneider M. Endoscopic removal of cysticercal cysts within the fourth ventricle. Technical note. J Neurosurg. 1999; 91:340-345. [PubMed: 10433327]

65. Cudlip SA, Wilkins PR, Marsh HT. Endoscopic removal of a third ventricular cysticercal cyst. Br J Neurosurg. 1998; 12:452-454. [PubMed: 10070452]

66. Seiffert E, Dreier JP, Ivens S, et al. Lasting blood-brain barrier disruption induces epileptic focus in the rat somatosensory cortex. J Neurosci. 2004; 24:7829-7836. [PubMed: 15356194]

67. Phiel CJ, Zhang F, Huang EY, Guenther MG, Lazar MA, Klein PS. Histone deacetylase is a direct target of valproic acid, a potent anticonvulsant, mood stabilizer, and teratogen. J Biol Chem. 2001; 276:36734-36741. [PubMed: 11473107]

68. Beutler AS, Li S, Nicol R, Walsh MJ. Carbamazepine is an inhibitor of histone deacetylases. Life Sci. 2005; 76:3107-3115. [PubMed: 15850602] 


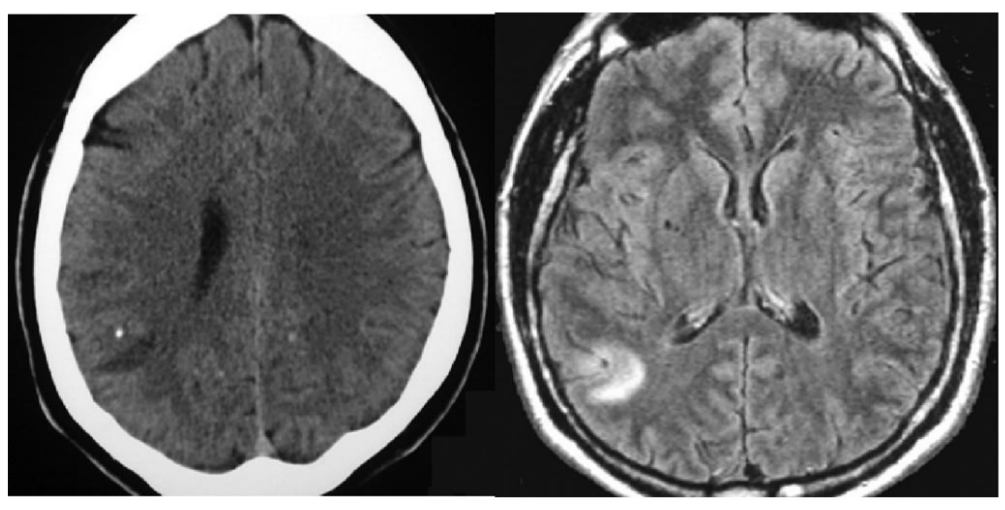

Figure 1.

Brain edema surrounding a calcified cyst as seen on CT (left) and MRI (right). 


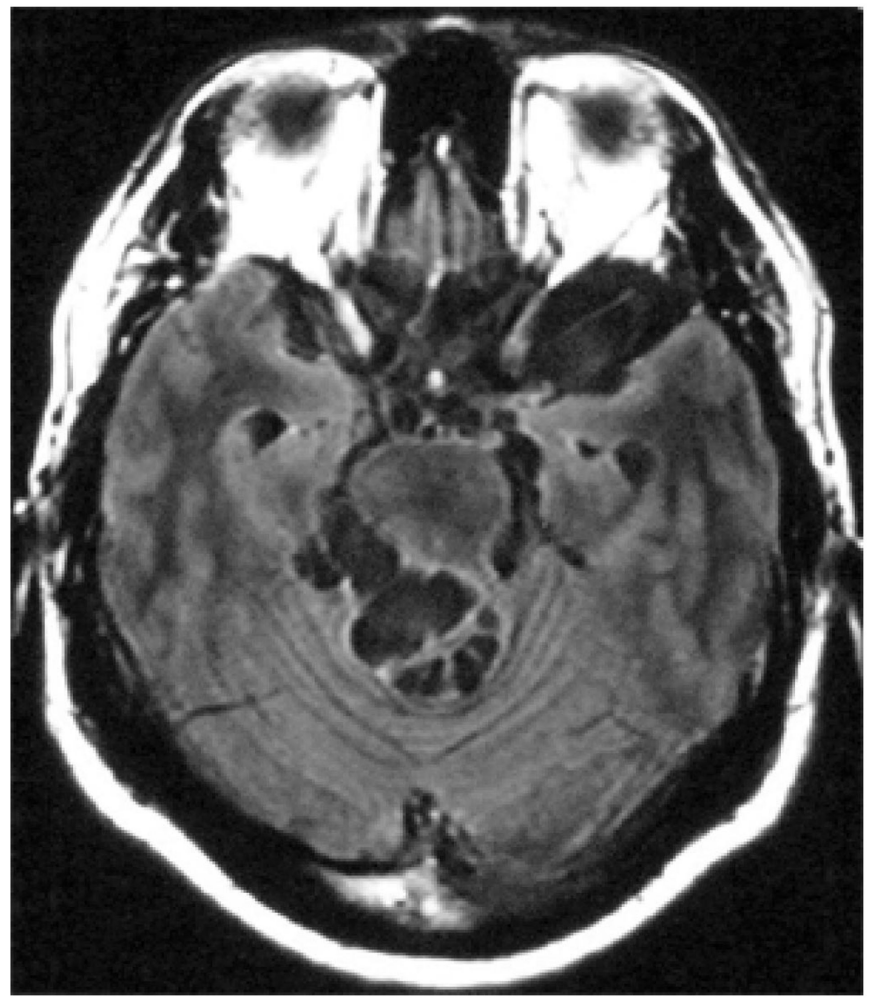

Figure 2.

Extraparenchymal (basal subarachnoid) cysticercosis. 